\title{
Reference Voltage Vector Based Model Predictive Torque Control with RMS Solution for PMSM
}

\author{
Chenwei Ma \\ Department of Electrical Energy, \\ Metals, Mechanical \\ Constructions \& Systems \\ Ghent University \\ EEDT-Decision and Control \\ Flanders Make \\ Ghent, Belgium \\ Institute of Electrical Engineering \\ Harbin Engineering University \\ Harbin, China \\ Chenwei.Ma@UGent.be \\ Xuliang Yao \\ Institute of Electrical Engineering \\ Harbin Engineering University \\ Harbin, China \\ yaoxuliang@hrbeu.edu.cn
}

\author{
Huayu Li \\ Department of Electrical Energy, \\ Metals, Mechanical \\ Constructions \& Systems \\ Ghent University \\ EEDT-Decision and Control \\ Flanders Make \\ Ghent, Belgium \\ Huayu.Li@UGent.be
}

\author{
Frederik De Belie \\ Department of Electrical Energy, \\ Metals, Mechanical \\ Constructions \& Systems \\ Ghent University \\ EEDT-Decision and Control \\ Flanders Make \\ Ghent, Belgium \\ Frederik.DeBelie@UGent.be
}

\begin{abstract}
To reduce the computational burden of a conventional model predictive torque controller (MPTC), a reference voltage vector based MPTC strategy is proposed. The reference voltage vector is obtained from the reference stator flux vector and the reference torque. According to the location of the reference voltage vector, a first optimal vector can be determined in a quite straightforward way, improving the system dynamic performance. Furthermore, in order to decrease the torque and flux ripple, a root mean square (RMS) based solution is employed to generate the reference voltage vector and calculate the duty ratio. This method aims at minimizing the RMS error of flux and torque during the whole control period. Then, the steady state performance is improved. Besides, since the new cost function contains only the reference voltage vector, the weighting factor in conventional MPTC is eliminated. In addition, to keep a balance between the steady state performance and switching frequency, the candidates for the second optimal vector are restricted to a certain scope. Simulations were carried out and the results verified the validation of the proposed MPTC strategy.
\end{abstract}

Keywords-permanent magnet synchronous motor (PMSM), model predictive torque control (MPTC), computational burden, torque and flux ripple

\section{INTRODUCTION}

Due to the advantages of high efficiency and high power density, permanent magnet synchronous motors (PMSMs) are brought into play in many industrial fields [1]. This increased introduction also means that the control of PMSMs receive more and more attention. Between the many control strategies developed for PMSMs, the finite control set-model predictive control (FCS-MPC) is of interest, having an intuitive concept, a straightforward implementation and taking into account the machine nonlinearities. The model predictive torque control (MPTC), based on FCS-MPC, could be regarded as an improved strategy of the conventional direct torque control of PMSMs. As done in DTC, the MPTC estimates the stator flux vector (and torque) for the voltage vector applied, but through the FCS-MPC also predicts it for all possible input voltage vectors, based on a model of the PMSM to be controlled. Then, according to the evaluation of a predefined cost function, an optimal voltage vector is searched for that minimizes this cost function. Another attractive aspect of MPTC is that variable constraints can be added to the model as well as cost function [2-4].

On the other hand, MPTC has the drawback of DTC in having a high torque and flux ripple, as a single voltage vector is applied during a control period. To address this problem, twovector based MPTC approaches have been proposed. In a first step of such a control strategy, an active vector vector is determined, based on the cost function evaluation [5]. The time duration of this first active voltage vector is calculated according to some rules. Then, a zero voltage vector is applied during the remaining control period. However, a zero vector may not be the optimal vector. Moreover, after inserting a zero voltage vector, it could be non-optimal to apply a new active voltage vector. As a result, novel two-vector strategies have been developed using an arbitrary vector as the second vector, resulting in more possibilities to control the PMSM [6]. Although such strategy can improve drive performance, the computational burden has remarkably increased as a result of the cost function to be evaluated for a higher amount of possible supply voltage vectors. In addition, also the time duration has to be determined. An increased computational effort and corresponding time delay could hamper the development of a higher dynamic drive performance in practical application. The problem is more severe, as recent development efforts on the power supply try to increase the switching frequency and hence lower the time available to perform calculations.

To reduce the computational burden, a modified MPTC strategy has been proposed in [7-8]. The reference torque and reference stator flux vector are converted to a reference voltage vector [7-8], based on deadbeat solution. It successfully forces the torque and stator flux vector to reach their reference values at the end of every control period. However, additional research effort is required to reduce the mean error of torque and flux. Moreover, the duty ratios of the two succeeding voltage vectors are determined based on tracking and minimizing the instantaneous voltage vector error. Besides, it should also guarantee a good global tracking performance of torque and flux. Even though the computational burden has decreased under 
this novel approach in the two-vector control strategy, additional improvements could be made to the steady state performance.

For this, in this paper, an improved reference voltage vector based MPTC is proposed, using a root mean square (RMS) solution. This strategy not only tries to reduce the amount of calculations in conventional enumerated MPTC but also further improves the torque and flux performance on a mean and global time scale. In the proposed strategy, a reference voltage vector will be generated according to the reference torque and reference stator flux. Based on the location of the reference voltage vector, a first optimal active vector can be computed. To reduce the torque and flux ripple, a two vector based control approach will be described. In such method, a second vector will be selected based on the selection of the first vector, in which the cost function evaluations for each of the vector combinations will be avoided. This strategy will reduce the computational burden further. The reference voltage vector generation and the duty ratio optimization are both based on a RMS torque and flux error minimization scheme. The goal of the optimization is to improve the steady state performance by lowering the flux and torque ripples. In addition, a new cost function, which contains only one variable being the reference voltage vector, is proposed. By using a single variable in the cost function, the weighting factor tuning in conventional MPTC can be avoided. Simulation results show the effectiveness of the proposed strategy.

\section{PMSM EQUATIONS AND INVERTER MODEL}

The synchronous reference frame is employed in this paper to analyze MPTC strategy. In such frame, the equations of the surface mounted PMSM ( $L_{d}=L_{q}=L_{s}$ ) in d-q reference frame can be expressed as follows:

$$
\begin{gathered}
V_{s d}=R_{s} I_{s d}+\frac{d \psi_{s d}}{d t}-\omega_{r} \psi_{s q} \\
V_{s q}=R_{s} I_{s q}+\frac{d \psi_{s q}}{d t}+\omega_{r} \psi_{s d} \\
\psi_{s d}=L_{s} I_{s d}+\psi_{f} \\
\psi_{s q}=L_{s} I_{s q} \\
T_{e}=\frac{3}{2} p \psi_{f} I_{s q}
\end{gathered}
$$

where $\psi_{s d}$ and $\psi_{s q}$ are d-axis and q-axis stator flux respectively, $V_{s d}$ and $V_{s q}$ are d-axis and q-axis stator voltage respectively, $I_{s d}$ and $I_{s q}$ are d-axis and q-axis stator current respectively, $T_{e}$ is electromagnetic torque, $p$ is the number of pole pairs, $R_{S}$ is stator resistance, $L_{s}$ is inductance, $\omega_{r}$ is electrical rotor speed, $\psi_{f}$ is permanent magnet flux linkage, assumed to be constant.

To drive the PMSM, a two-level voltage source inverter (VSI) is utilized in this paper, shown in Fig. 1. The voltage vectors generated by the inverter, shown in Fig. 2, can be expressed as follow:

$$
V_{j}=\frac{2}{3} V_{d c}\left(S_{a}+e^{i 2 \pi / 3} S_{b}+e^{i 4 \pi / 3} S_{c}\right)
$$

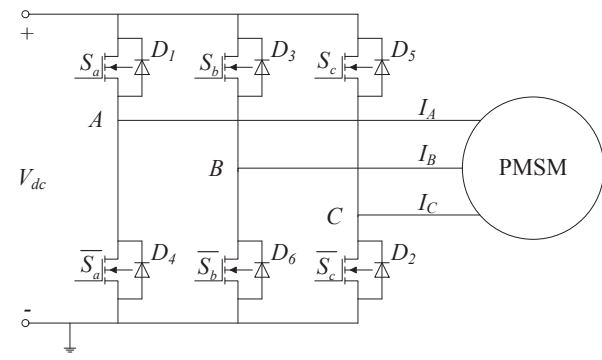

Fig. 1. Two level VSI.

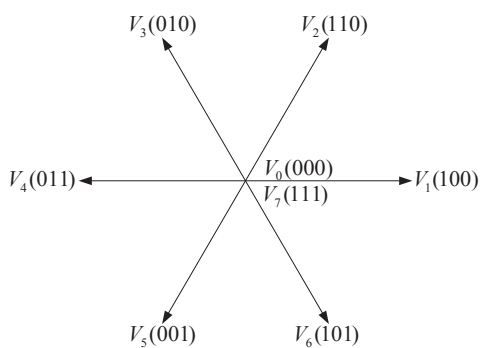

Fig. 2. Possible switching states of two level VSI.

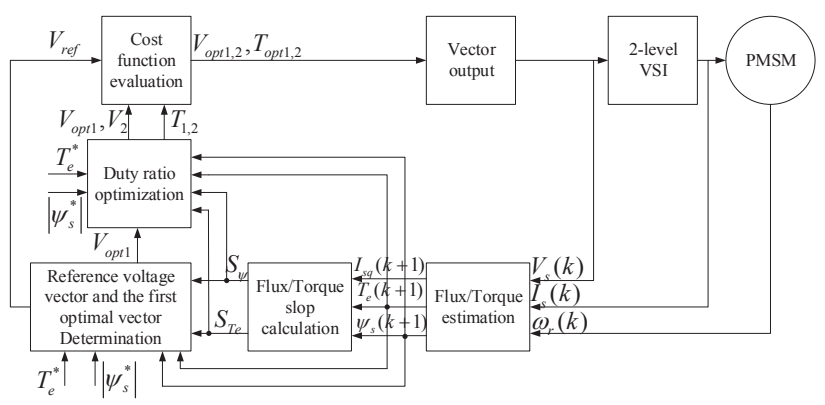

Fig. 3. Diagram of the proposed MPTC strategy.

where $V_{j}(j=0, \ldots, 7)$ are the voltage vectors, $S_{a}, S_{b}, S_{c}$ are the switching states of the three inverter legs.

\section{Reference Voltage Vector Based MPTC StRategy}

Fig. 3 shows the control diagram of the proposed MPTC strategy. It starts with the flux and torque estimation, then the flux and torque time gradient calculation following the construction of the reference voltage vector. It is followed by the duty ratio optimization and the final step that includes the optimal vector determination, based on a cost function evaluation. All these steps will be discussed hereafter.

\section{A. Stator Flux and Torque Estimation}

In a discrete time implementation, the optimal voltage vector computed is to be applied during the next control period. This means that the stator flux vector and torque at the end of the current control period, being also the start of next control period, have to be estimated based on the voltage vector as computed in the previous control period. According to the PMSM model, the stator flux and torque at the start of the next control period $(\mathrm{k}+1)$ can be estimated as:

$$
\psi_{s}(k+1)=\psi_{s}(k)+T_{1}(k) V_{s 1}(k)+T_{2}(k) V_{s 2}(k)-T_{s} R_{s} I_{s}(k)
$$




$$
\begin{gathered}
T_{e}(k+1)=\frac{3}{2} p \psi_{f} I_{s q}(k+1) \\
I_{s q}(k+1)=I_{s q}(k)+\frac{T_{s}}{L_{s}}\left[-L_{s} \omega_{r} I_{s d}(k)-R_{s} I_{s q}(k)-\omega_{r} \psi_{f}\right] \\
+\frac{1}{L_{s}}\left[T_{1}(k) V_{s q 1}(k)+T_{2}(k) V_{s q 2}(k)\right]
\end{gathered}
$$

where $\psi_{s}=\left[\psi_{s d}, \psi_{s q}\right]^{T}, I_{s}=\left[I_{s d}, I_{s q}\right]^{T}$ are the values at the start of a control period, $V_{s}=\left[V_{s d}, V_{s q}\right]^{T}, T_{s}$ is the total control period, $V_{s 1}$ and $V_{s 2}$ are the optimal voltage vectors, constant in time and applied during the first interval $T_{1}$ and second interval $T_{2}$ respectively. It should be noted that $T_{1} \leq T_{s}$ and $T_{2}=T_{s}-T_{1}$.

\section{B. Flux and Torque Gradients}

The voltage vector is a unique input variable for VSI driven PMSM. On the other hand, flux and torque are the most important state variables in MPTC. As such, it is of interest to deduce the variations of flux and torque under the input voltage vector applied. In the proposed MPTC strategy, the relationships are significant for the reference voltage vector determination and duty ratio optimization. According to the machine model in (1)-(4), the flux derivative during a voltage vector supply can be represented as:

$$
\frac{d\left|\psi_{s}\right|}{d t}=\frac{\psi_{s} \cdot\left(V_{s}-R I_{s}\right)}{\left|\psi_{s}\right|}
$$

As the control period is $T_{s}$, the flux variation due to the applied voltage vector can be expressed as:

$$
\Delta\left|\psi_{s}\right|=\frac{\psi_{s} \cdot\left(V_{s}-R I_{s}\right)}{\left|\psi_{s}\right|} T_{s} \triangleq S_{\psi} T_{s}
$$

where $S_{\psi}=\frac{\psi_{s} \cdot\left(V_{s}-R I_{s}\right)}{\left|\psi_{s}\right|}$ represents the flux slope under the voltage vector $V_{s}$.

Similarly, based on (1)-(5), the torque derivative and the torque variation under the voltage vector can be deduced as:

$$
\begin{gathered}
\frac{d T_{e}}{d t}=\frac{3 p \psi_{f}}{2 L_{s}}\left(V_{s q}-R_{s} I_{s q}-\omega_{r} \psi_{s d}\right) \\
\Delta T_{e}=\frac{3 p \psi_{f}}{2 L_{s}}\left(V_{s q}-R_{s} I_{s q}-\omega_{r} \psi_{s d}\right) T_{s} \triangleq S_{T e} T_{s}
\end{gathered}
$$

respectively, $S_{T e}=\frac{3 p \psi_{f}}{2 L_{s}}\left(V_{s q}-R_{s} I_{s q}-\omega_{r} \psi_{s d}\right)$ is the torque gradient under the voltage vector $V_{s}$. Since $T_{s}$ is short enough to state that all the variables are with small variation and therefore approximated by constants within one control period, both flux and torque gradients can be regarded as constants.

\section{RMS-Based Reference Voltage Vector Prediction}

Now that the torque and flux vector can be estimated, and the torque and flux gradients can be computed, a reference

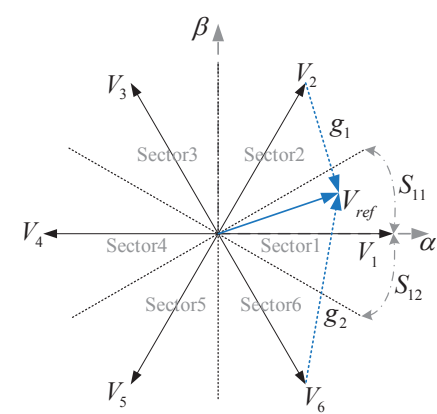

Fig. 4. Principle diagram of vector selection.

voltage can be determined that minimizes the RMS error against set values and gradients of flux and torque.

In a conventional two-vector MPTC strategy, the predictions of flux and torque, as well as the duty ratio optimizations have to be conducted for all possible vector combinations. In [6], the first and the second vector are all arbitrary vectors, which provides $7 \times 7=49$ possible vector combinations. Although the steady state performance is improved, many calculations are required. In [7], the second vector is selected in a border scope and it provides a reduced amount of 18 possibilities instead of 49 , reducing the computational burden. However, considering that the duty ratio is also to be estimated for all vector combinations, the computational cost is still significant. As an alternative method against considering all possible inputs, in [8], a reference voltage vector method is utilized. It aims at forcing the torque and flux equal to the reference values at the end of each control period and relies on a deadbeat solution for the voltage to be applied, referred to as the reference voltage vector. Hence, this strategy of using a reference voltage reduced the computational burden further.

In this paper, the previous method is further improved by providing a RMS-based reference voltage vector prediction. In this advanced method, a reference voltage vector is used that allows minimizing the RMS error of the flux and torque. It aims at improving the steady state performance by reducing the torque and flux ripples. On the other hand, in conventional MPTC, a weighting factor is required to adjust the weightings of flux and torque in cost function. In the proposed method, the weighting factor can be omitted, since the reference flux and reference torque are converted to reference voltage vector.

The RMS errors of the flux and torque can be expressed as:

$$
\begin{aligned}
& E_{\psi}=\frac{1}{T_{s}} \int_{0}^{T_{s}}\left(\psi_{s}^{e}+s_{\psi} t\right)^{2} d t \\
& E_{T e}=\frac{1}{T_{s}} \int_{0}^{T_{s}}\left(T_{e}^{e}+s_{T e} t\right)^{2} d t
\end{aligned}
$$

respectively, where $\psi_{s}^{e}=\psi_{s}(k+1)-\psi_{s}^{*}, T_{e}^{e}=T_{e}(k+1)-T_{e}^{*}$ are the initial flux and torque errors at the beginning of the next control period respectively. It should be noticed that to compensate the one step delay, $(\mathrm{k}+1)$ th variables are used for (14) and (15). According to the RMS principle, based on (16), a reference voltage vector can be deduced which solution is given in (17)-(18) 


$$
\begin{gathered}
\left\{\begin{array}{l}
\frac{d E_{\psi}}{d V_{s}}=0 \\
\frac{d E_{T e}}{d V_{s}}=0
\end{array}\right. \\
V_{\text {ref }}=\left[\begin{array}{l}
V_{s d}(k+1) \\
V_{s q}(k+1)
\end{array}\right]
\end{gathered}
$$

where

$$
\left\{\begin{array}{l}
V_{s d}(k+1)=-\frac{3 \psi_{s}^{e}\left|\psi_{s}(k+1)\right|}{2 T_{s} \psi_{s d}(k+1)}+\frac{R_{s} I_{s}(k+1) \bullet \psi_{s}(k+1)}{\psi_{s d}(k+1)}-\frac{\psi_{s q}(k+1) V_{s q}(k+1)}{\psi_{s d}(k+1)} \\
V_{s q}(k+1)=I_{s q}(k+1)\left(R_{s}+\omega_{r} L_{s}\right)-\frac{L_{s} T_{e}^{e}}{T_{s} p \psi_{f}}
\end{array}\right.
$$

In the other way around, by applying the reference voltage vector in (17) during the $(\mathrm{k}+1)$ th control period, it is tried to minimize the RMS errors of the flux and torque during this control period. To generate the reference voltage, two voltage vectors are applied during each control period. The first vector is selected from the six nonzero vectors being the active vectors. For the second vector, the two adjacent vectors of the first vector as well as the zero vector with the least amount of switching actions are considered. Hence, the first voltage vector should be firstly determined. For this purpose, the $\alpha-\beta$ reference frame is divided into six sectors of sixty degrees, as shown in Fig. 4. Each sector contains a single active or nonzero vector. The first vector to be applied is determined by the sector that holds the reference voltage vector. For instance, in Fig. 4, according to the phase angle of the reference voltage vector, the reference voltage vector is located in sector 1 , and hence the vector $V_{1}$ will be the first optimal voltage vector.

\section{Duty Ratio Optimization}

After getting the first optimal voltage vector, the candidates for the second optimal vector can be identified as mentioned before. Then the duty ratio calculation is the main task, resulting in the time duration to apply the first vector. In [6], the duty ratio optimization is based on a deadbeat torque control, which does not take the tracking of flux into account. In [8], the principle employed to determine the duty ratio is minimizing the tracking error of reference voltage vector, while the reference voltage vector is obtained based on deadbeat solution. Thus, this method of duty ratio optimization aims at nullifying the tracking error of flux and torque at the end of control period only. In this paper, to be consistent with the reference voltage vector prediction and to improve the steady state performance, the RMS solution is used to determine the duty ratio. The RMS error of flux and torque can be expressed as [9]:

$$
\begin{aligned}
E= & \frac{\lambda_{\psi}}{T_{s}} \int_{0}^{T_{1}}\left(\psi_{s}^{e}+s_{\psi 1} t\right)^{2} d t+\frac{\lambda_{\psi}}{T_{s}} \int_{T_{1}}^{T_{s}}\left[\psi_{s}^{e}+s_{\psi 1} t+s_{\psi 2}\left(t-T_{1}\right)\right]^{2} d t \\
& +\frac{1}{T_{s}} \int_{0}^{T_{1}}\left(T_{e}^{e}+s_{T e 1} t\right)^{2} d t+\frac{1}{T_{s}} \int_{T_{1}}^{T_{s}}\left[T_{e}^{e}+s_{T e 1} t+s_{T e 2}\left(t-T_{1}\right)\right]^{2} d t
\end{aligned}
$$

where $\lambda_{\psi}$ is the weighting factor of stator flux, $S_{\psi 1}$ and $S_{\psi 2}$ are the flux gradients for the first vector and the second vector candidate respectively, $S_{T e 1}$ and $S_{T e 2}$ are the torque gradients for the first vector and the second vector candidate respectively. Hence, letting $d E / d T_{1}=0$, the time duration for the first voltage vector can be expressed as:

$$
T_{1}=\frac{-B+\sqrt{B^{2}-4 A C}}{2 A}
$$

where

$$
\begin{gathered}
A=-\lambda_{\psi} s_{\psi}^{\prime}\left(s_{\psi 1}+s_{\psi}^{\prime}\right)-s_{T e}^{\prime}\left(s_{T e 1}+s_{T e}^{\prime}\right) \\
B=2 \lambda_{\psi} s_{\psi}^{\prime}\left(T_{s} s_{\psi}^{\prime}-\psi_{s}^{e}\right)+2 s_{T e}^{\prime}\left(T_{s} s_{T e}^{\prime}-T_{e}^{e}\right) \\
C=T_{s} \lambda_{\psi} s_{\psi}^{\prime}\left(T_{s} s_{\psi 2}+2 \psi_{s}^{e}\right)+T_{s} s_{T e}^{\prime}\left(T_{s} s_{T e 2}+2 T_{e}^{e}\right)
\end{gathered}
$$

and $S_{\psi}^{\prime}=S_{\psi 1}-S_{\psi 2}, S_{T e}^{\prime}=S_{T e 1}-S_{T e 2}$. Duty ratio should be optimized for the three vector combinations, and the optimal vector combination is determined according to cost function.

\section{E. Cost Function Evaluation}

In conventional MPTC, a weighting factor is required to adjust the weightings of flux and torque in cost function. In the proposed method, the weighting factor can be omitted, since the reference flux and reference torque are converted to reference voltage vector. The new cost function can be denoted as:

$$
g=\left|V_{r e f}-d_{1} V_{s 1}-\left(1-d_{1}\right) V_{s 2}\right|
$$

where $d_{1}=T_{1} / T_{s}$ is the duty ratio of the first vector, determined by the previous step. As aforementioned, after determining the first optimal vector, three candidates should be evaluated by this cost function in order to obtain the second optimal vector. However, in this strategy, only two candidate vectors out of three have to be evaluated according to the location of the reference voltage vector. For example, if the reference voltage vector locates in region $S_{11}$ of sector 1, as shown in Fig. 4, only the nonzero vector $V_{2}$ and zero vector $V_{0}$ need to be evaluated according to the cost function. It is because the amplitude error between $V_{2}$ and $V_{\text {ref }}\left(g_{1}=\left|V_{2}-V_{\text {ref }}\right|\right)$ is smaller than that between $V_{6}$ and $V_{\text {ref }}\left(g_{2}=\left|V_{6}-V_{\text {ref }}\right|\right)$. Similarly, in case the reference voltage vector locates in $S_{12}$ of sector 1 , only $V_{6}$ and $V_{0}$ have to be evaluated. In the special case of a reference voltage vector located on the middle line of sector 1 , the amplitude error between $V_{2}$ and $V_{\text {ref }}$ equals the one between $V_{6}$ and $V_{\text {ref }}\left(g_{1}=g_{2}\right)$. In that case, either $V_{2}$ or $V_{6}$ should be selected, preferably in the direction of rotation.

\section{Simulation Results}

To verify the validation of the proposed MPTC, some comparative simulations are conducted in Matlab/Simulink. The parameters of the simulated motor are listed in Table 1. Three two-vector MPTC strategies, which are the conventional twovector MPTC with torque deadbeat solution (MPTC-DB) [5], reference voltage vector based MPTC with deadbeat solution (RVV-MPTC-DB) [8], and the proposed MPTC will be compared. Sampling frequency of the three strategies is $5 \mathrm{kHz}$.

Fig. 5 shows the waveforms of the torque, stator flux and phase current for three MPTC strategies. To show the dynamic performance of the strategies, the reference torque suddenly changes from $10 \mathrm{Nm}$ to $12 \mathrm{Nm}$ at $0.2 \mathrm{~s}$. It can be seen in Fig. 5(a) 
TABLE I. MOTOR PARAMETERS

\begin{tabular}{|c|c|c|}
\hline Motor parameter & & Value \\
\hline DC voltage & $V_{d c}$ & $200 \mathrm{~V}$ \\
\hline Number of pole pairs & $p$ & 1 \\
\hline Permanent magnet flux linkage & $\psi_{f}$ & $1 \mathrm{~Wb}$ \\
\hline Stator resistance & $R_{s}$ & $1.91 \Omega$ \\
\hline Inductance & $L_{s}$ & $0.016 \mathrm{H}$ \\
\hline Flux amplitude reference & $\left|\psi_{s}^{*}\right|$ & $1.0227 \mathrm{~Wb}$ \\
\hline
\end{tabular}

TABLE II. COMPARATION OF STEADY STATE PERFORMANCE

\begin{tabular}{|c|c|c|c|}
\hline Parameter & MPTC-DB & $\begin{array}{c}\text { RVV- } \\
\text { MPTC-DB }\end{array}$ & $\begin{array}{c}\text { Proposed } \\
\text { MPTC }\end{array}$ \\
\hline$T_{\text {rip }}$ & $0.1494 \mathrm{Nm}$ & $0.1249 \mathrm{Nm}$ & $0.0772 \mathrm{Nm}$ \\
\hline$\psi_{\text {rip }}$ & $0.00795 \mathrm{~Wb}$ & $0.00101 \mathrm{~Wb}$ & $0.00102 \mathrm{~Wb}$ \\
\hline
\end{tabular}

that the MPTC-DB strategy can reduce the torque ripple. However, the torque can reach the reference value at the end of control period only, since a torque deadbeat principle is utilized to calculate the duty ratio. Besides, the tracking performance of stator flux is not ideal, since flux error is not included in duty ratio optimization. For RVV-MPTC-DB strategy, the reference voltage vector is converted from the reference flux and reference torque. The result is shown in Fig. 5(b). It follows that the steady state of the stator flux is better than that of the MPTC-DB strategy shown before in Fig. 5(a). However, since the reference voltage vector generation is also based on deadbeat solution, while the duty ratio optimization is based on tracking error minimization of the reference voltage vector, the control performance of torque is similar with the MPTC-DB strategy. Furthermore, a torque fluctuation can be noticed in Fig. 5(b). In the proposed strategy, RMS principle is introduced to reference voltage generation and duty ratio optimization. Thus, in Fig. 5(c), the steady state performance of the torque and flux under the proposed MPTC strategy is much better than other two strategies. Instead of forcing the torque to reach its reference value at the end of control period, the RMS error of the flux and torque is minimized in the proposed MPTC strategy. Therefore, reduction of the torque and flux ripple is achieved.

Fig. 6 shows the tracking trajectories of torque and flux under the three MPTC strategies. For the torque tracking trajectory, it can be seen in Fig. 6(a) and Fig. 6(b) that the performance of MPTC-DB strategy and RVV-MPTC-DB strategy are similar, despite the torque fluctuation in RVVMPTC-DB strategy. It can be easily seen in Fig. 6(c) that the proposed MPTC strategy performs best in torque tracking among the three strategies. The mean error of torque is minimized, which leads to lower torque ripples than the other two strategies. On the other hand, RVV-MPTC-DB and the proposed MPTC strategy present better performance in flux error tracking than MPTC-DB strategy. A comparison of the steady state performance in terms of torque and stator flux ripple for the three strategies is presented in Table 2. The torque and flux ripple are calculated according to the following equations:

$$
\left\{\begin{array}{l}
T_{\text {rip }}=\sqrt{\frac{1}{n} \sum_{i=1}^{n}\left(T_{e}(i)-T_{e}^{*}\right)^{2}} \\
\psi_{\text {rip }}=\sqrt{\frac{1}{n} \sum_{i=1}^{n}\left(\psi_{s}(i)-\psi_{s}^{*}\right)^{2}}
\end{array}\right.
$$
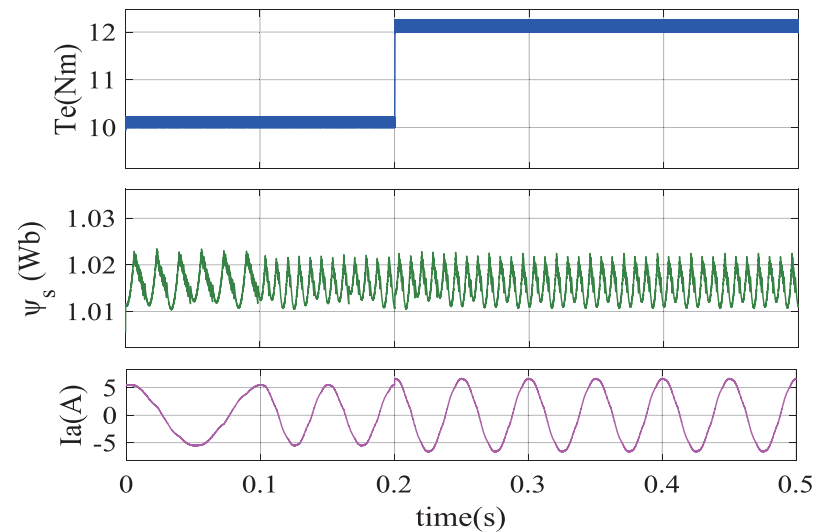

(a)

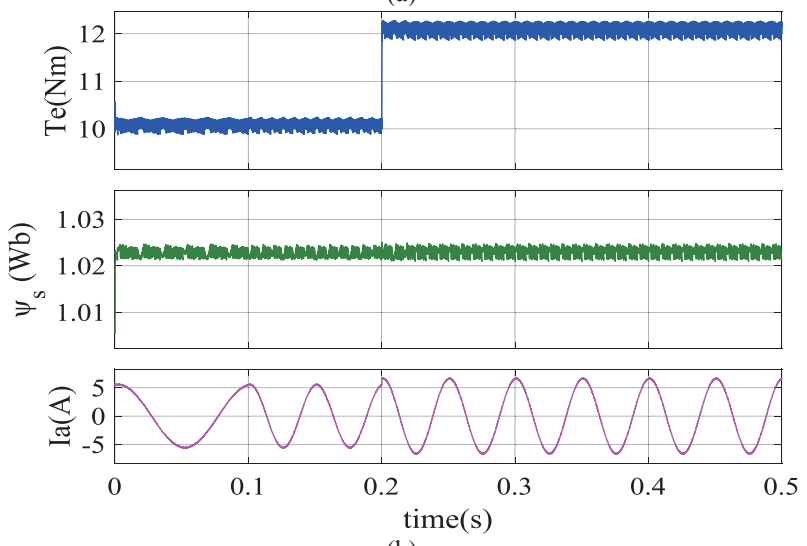

(b)
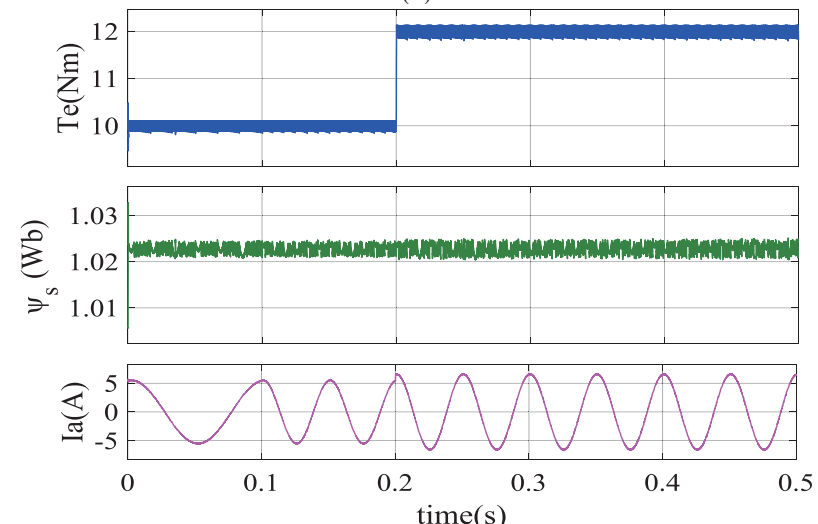

(c)

Fig. 5. Waveforms of torque, stator flux and phase current. (a) MPTCDB. (b) RVV-MPTC-DB (c). Proposed MPTC.

where $n$ denotes the sampling number.

It can be observed in Fig. 7 that the proposed MPTC strategy can achieve better current harmonic spectrum, which further demonstrates the good steady state performance of the proposed MPTC. Besides, the switching states of the three strategies are shown in Fig. 8. It can be seen that the RMS based reference voltage vector and duty ratio optimization can lead to different switching states, which result in better steady state performance. In addition, compared to the convention enumerated MPTC-DB strategy, the amount of evaluations and additional computations 


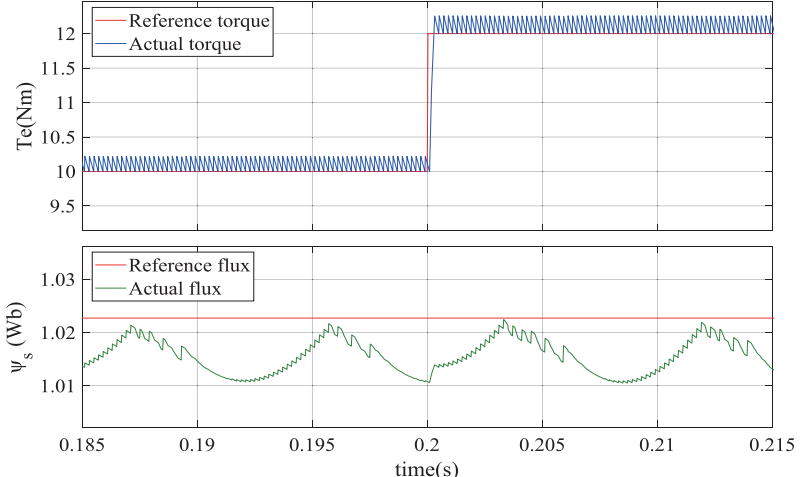

(a)
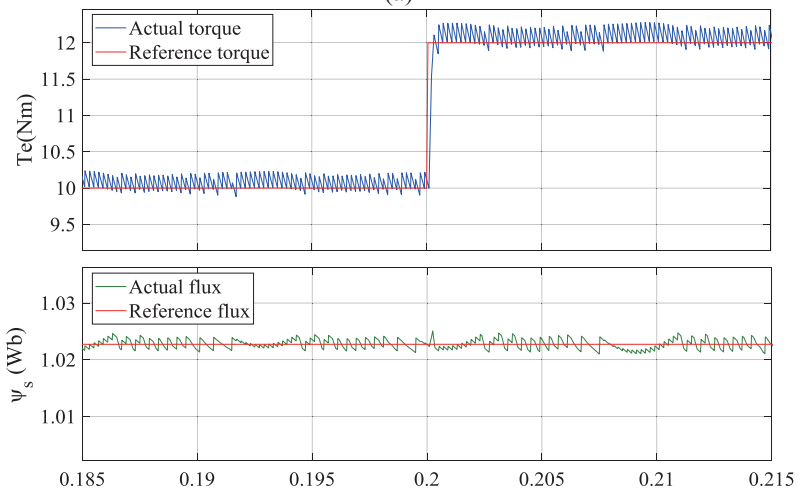

(b)
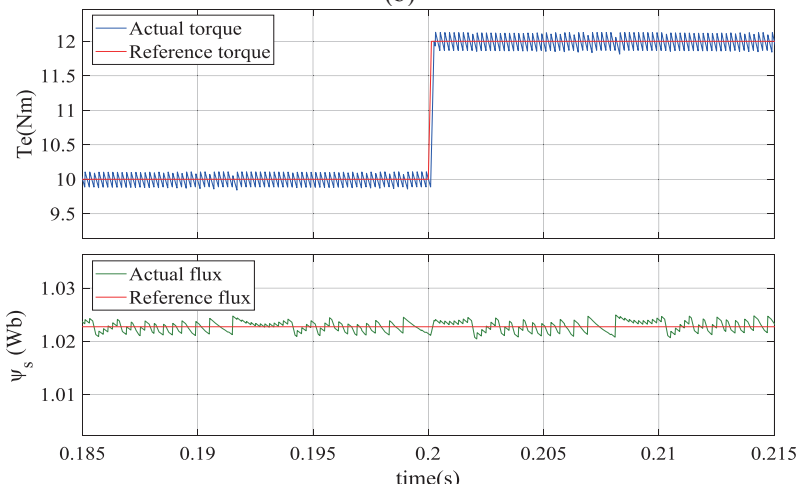

(c)

Fig. 6. Tracking trajectories of torque and stator flux. (a) MPTC-DB. (b) RVV-MPTC-DB (c). Proposed MPTC.

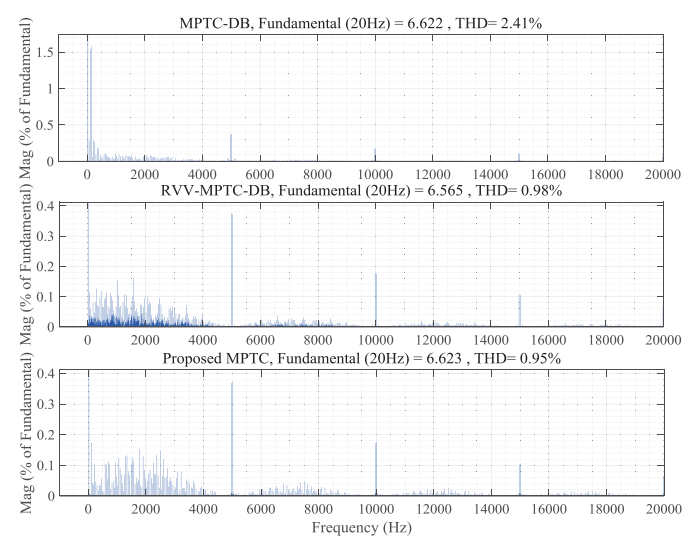

Fig. 7. Comparison of harmonic spectrum of phase current

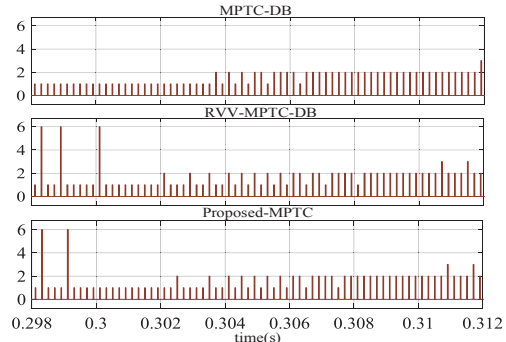

Fig. 8. Switching states of the three strategies

in the proposed MPTC are reduced and the tuning work of weighting factor in cost function can be omitted.

\section{CONLUSION}

This paper proposes an improved reference voltage vector based MPTC for PMSM drives. Using a RMS based solution to reduce flux and torque ripples, a novel reference voltage vector is generated, based on the reference stator flux and reference torque. From this reference, vectors and time intervals for the two-vector control approach are selected in an optimal way. Compared to the finite control set methods, less input possibilities are considered during the optimization step so that the computational burden can be reduced. In addition, the new cost function to select the optimal voltage vector from a reduced amount of possible voltage vectors, uses no weighting factors and avoids the corresponding tuning work.

\section{ACKNOWLEDGMENT}

This research was partially supported by Flanders Make, the strategic research centre for the manufacturing industry through the Multisystem Learning Control project.

\section{REFERENCES}

[1] P. Kakosimos and H. Abu-Rub, "Predictive Speed Control With Short Prediction Horizon for Permanent Magnet Synchronous Motor Drives," IEEE Trans. Power Electron., vol. 33, no. 3, pp. 2740-2750, 2018.

[2] M. Amiri, J. Milimonfared, and D. A. Khaburi, "Predictive Torque Control Implementation for Induction Motors Based on Discrete Space Vector Modulation,” IEEE Trans. Ind. Electron., vol. 65, no. 9, pp. 68816889, 2018.

[3] M. Siami, D. Arab Khaburi, and J. Rodriguez, "Simplified Finite Control Set-Model Predictive Control for Matrix Converter-Fed PMSM Drives,' IEEE Trans. Power Electron., vol. 33, no. 3, pp. 2438-2446, 2018.

[4] Y. Cho, Y. Bak, and K.-B. Lee, "Torque-Ripple Reduction and Fast Torque Response Strategy for Predictive Torque Control of Induction Motors," IEEE Trans. Power Electron., vol. 33, no. 3, pp. 2458-2470, 2018.

[5] Y. Xu, Q. Zhou and B. Zhang, "A Model Predictive Torque Control Strategy of PMSM with Torque Deadbeat Duty Cycle Control," IEEE Int. Power. Elec., 2016, pp. 782-785.

[6] Y. Zhang and H. Yang, "Generalized Two-Vector-Based ModelPredictive Torque Control of Induction Motor Drives," IEEE Trans. Power Electron., vol. 30, no. 7, pp. 3818-3829, 2015.

[7] Y. Zhang and H. Yang, "Two-Vector-Based Model Predictive Torque Control Without Weighting Factors for Induction Motor Drives," IEEE Trans. Power Electron., vol. 31, no. 2, pp. 1381-1390, 2016.

[8] X. Zhang and B. Hou, "Double Vectors Model Predictive Torque Control Without Weighting Factor Based on Voltage Tracking Error," IEEE Trans. Power Electron., vol. 33, no. 3, pp. 2368-2380, 2018.

[9] F. Niu, K. Li, and Y. Wang, "Direct Torque Control for PermanentMagnet Synchronous Machines Based on Duty Ratio Modulation," IEEE Trans. Ind. Electron., vol. 62, no. 10, pp. 6160-6170, 2015. 